\title{
Defects Caused by Chain Ends in Polymer Crystals and Interpretation of Plastic Deformation and $\gamma$-Relaxation in Terms of Them
}

\author{
Masahiro Matsui, * Ryōhei Masui, and Yasaku WAdA \\ Department of Applied Physics, Faculty of Engineering, University \\ of Tokyo, Bunkyo-ku, Tokyo, Japán.
}

(Received August 17, 1970)

\begin{abstract}
Strain energy of various types of defects caused by chain ends in the polymer crystal is theoretically estimated by using interchain and intrachain potentials of polyethylene. It is concluded that screw dislocations running through chain ends have minimum energy when chain ends are not so close to the crystal surface. For the end close to the surface, on the other hand, a vacancy row from the end to the surface is proved to be the most stable and the minimum distance of the end from the surface for screw dislocation formation is $c a$. $30 \AA$ for linear polyethylene of usual molecular weight. The crystal is expected to have a mosaic structure owing to screw dislocations and vacancy rows.

Transformation of orthorhombic crystal to monoclinic and $\{110\}$ twinning, both observed in the early stage of plastic deformation of polyethylene, are interpreted in terms of the vacancy rows and screw dislocations described above. The $\gamma$-relaxation of as-grown polyethylene crystals is explained by thermal motion of screw dislocations over the Peierls potential and a satisfactory agreement with experiment is attained by the two-state model theory for both relaxation strength and activation energy, by use of reasonable estimates for parameters in the theory.
\end{abstract}

KEY WORDS Defect/Dislocation/Chain End/Crystal/Polyethylene/ Poly(oxymethylene)/Twinning/Phase Transformation/ $\gamma$-Relaxation/

Plastic Deformation/

As-grown polymer crystals include numerous defects of various types. The defects form because the crystal grows through successive attachment of folded chain molecules with which disorders in chemical structure, i.e., branches, disorders in tacticity, chain ends, and others, are introduced into the crystal. Some of them might be outside the crystal and constitute the noncrystalline phase on the surface of crystals. However, crystals with defects have lower energy than the noncrystalline phase and consequently a considerable number of disorders may be present within the crystal.

According to Keller and Priest, ${ }^{11}$ about onetenth of the chain ends in polyethylene single crystals are located within the crystal so that the ends are highly resistant to oxidation. Various types of defects caused by chain ends have been

\footnotetext{
* Present address: Teijin Ltd., Matsuyama Plant, Matsuyama, Japan.
}

suggested by Predecki and Statton. ${ }^{2}$ According to them, screw dislocations and edge dislocations are expected to run through chain ends perpendicularly and in parallel with the chain axis, respectively.

In the first part of this paper, results of calculations will be presented for the strain energy of possible types of defects caused by chain ends, and the most stable types will be suggested.

In the second part, mechanisms of phase transformation and twinning which are observed in the early stage of plastic deformation of polyethylene single crystals ${ }^{3}$ are interpreted in terms of defects predicted in the first part of this paper.

Finally, a dislocation theory will be presented for the $\gamma$-relaxation of polyethylene and other highly crystalline polymers. The $\gamma$-relaxation of polyethylene consists at least of two peaks., The low temperature peak, designated $\gamma_{1}$ in this 
paper, has been ascribed by various authors ${ }^{4,5}$ to defects in the crystal. In the present study, the peak height and activation energy of the $\gamma_{1}$-peak will be predicted in terms of thermal motion of screw dislocations caused by chain ends.

\section{INTERCHAIN POTENTIAL}

In this study, mainly polyethylene crystals are treated as a typical example of polymer crystals. In the orthorhombic polyethylene crystal, a chain is surrounded by six neighbors. In the following, an equal chain separation is assumed in each direction for simplicity, i.e., the crystal is approximated by hexagonal symmetry. The chain separation $l^{*}$ is taken as $4.60 \AA$ at the average.

In determining the intermolecular interaction, chains of polyethylene are assumed to be a linear arrangement of units $\left(\mathrm{CH}_{2}\right.$-groups) with equal distance of $c / 2$. The intermolecular potential is taken as the sum of the pair-wise potential between units, which is expressed by the Lennard-Jones 6-12 potential,

$$
u(r)=-2 \varepsilon\left(\frac{r^{*}}{r}\right)^{6}+\varepsilon\left(\frac{r^{*}}{r}\right)^{12}
$$

where $\varepsilon$ and $r^{*}$ are constants and $r$ is the distance between units.

Then the potential energy between a unit and a neighbor chain is (Figure 1a),

$$
w(l)=\int_{-\infty}^{\infty} u \rho \mathrm{d} z=-\frac{3 \pi}{4} \rho \varepsilon\left(\frac{r^{* 6}}{l^{5}}-\frac{21}{64} \frac{r^{* 12}}{l^{11}}\right)
$$

where $l$ is the distance of the chain from the

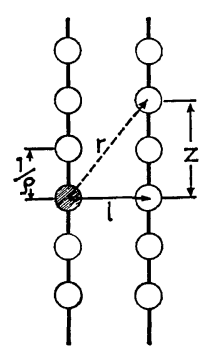

(a)

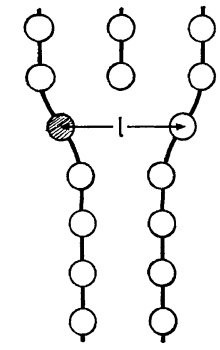

(b)
Figure 1. Models of chains for calculating interchain potential: (a), a unit and a neighboring regular chain; (b), a unit and a neighboring curved chain. unit, and $\rho$ the linear number density of units in the chain $(\rho=2 / c)$. By minimizing $w$ with respect to $l$, expressions of equilibrium values of $l$ and $w$ are obtained. By equaling these expressions to experimental lattice constant and two-sixths of lattice energy, $l^{*}=4.60 \AA$ and $\left|w^{*}\right|=(2 / 6) \times 1.84 \mathrm{kcal} / \mathrm{mol}$ unit, ${ }^{6}$ parameters $r^{*}$ and $\varepsilon$ are determined as $4.86 \AA$ and $6.62 \times 10^{-15} \mathrm{erg}$, respectively.

For distorted chains in the vicinity of defects, $l$ is not necessarily $l^{*}$ and chains may be locally curved. In this case, the potential energy $w(l)$ between a unit and a curved neighbor chain is assumed to be a function of $l$, which expresses the perpendicular distance between the unit and the chain (Figure 1b). This approximation may be acceptable because of the short-range nature of the interchain potential and the low curvature of the curved chain.

\section{INTRACHAIN POTENTIAL}

In determining the increase in intrachain potential on account of chain bending, the planar zigzag conformation of polyethylene molecules is taken into account because a strong anisotropy is expected for the bending.

Three types of bending as shown in Figure 2 are considered: type a bending perpendicular to zigzag plane, type $b$ parallel to the plane, and type $\mathrm{c}$ oblique to the plane. The first two occur successively in the $\{110\}$ plane and the last in the $\{200\}$ plane. Type a bending results from torsion of $\mathrm{C}-\mathrm{C}$ bonds and the force constant is the smallest. Type $b$ bending is brought about

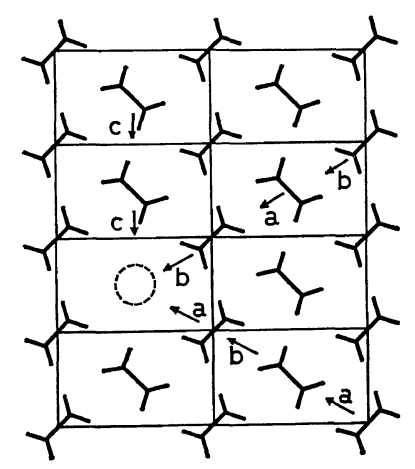

Figure 2. Three types of bending (a, b, and $c)$ of planar zigzag chains towards a vacancy from three directions in the orthorhombic polyethylene crystal. 

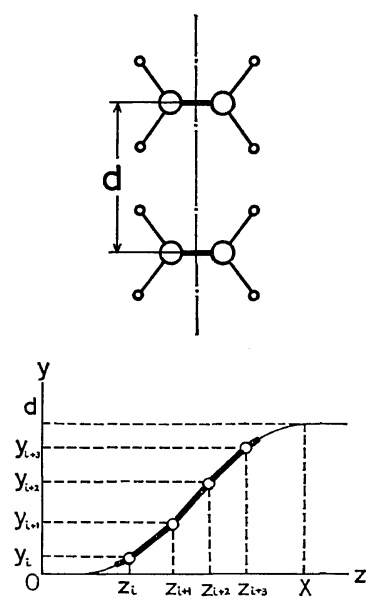

(a)
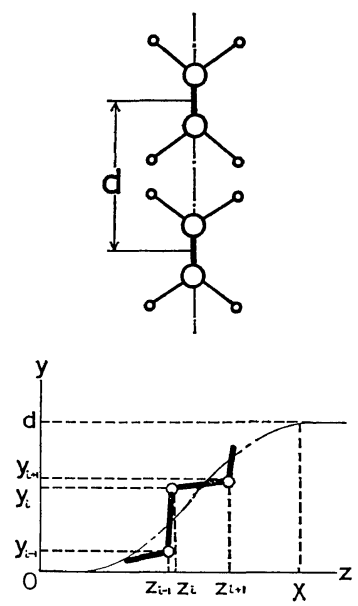

(b)
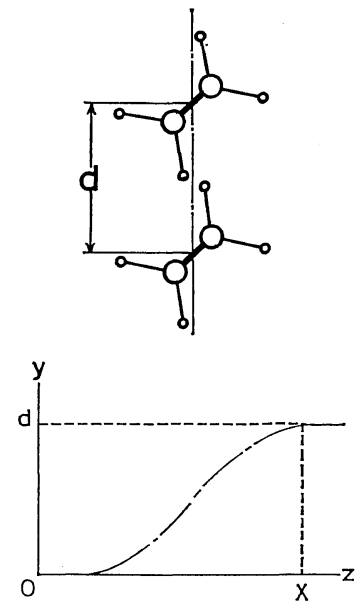

(c)

Figure 3. Models of chains for calculating intrachain potential of three types of bending: (a), bending perpendicular to zigzag plane; (b), parallel to zigzag plane; (c), oblique to zigzag plane.

by a bond-angle change with a large force constant. Type $\mathrm{c}$ bending is a composite of the two, having an intermediate force constant.

The increase in intrachain potential by bending, $\Delta U_{\text {intra }}$, depends on the shape of curved chain. Here we assume that the length of the curved part of a chain along the $z$-axis (chain-axis) is $X$ and the perpendicular shift in the bend plane ( $y z$-plane) is $d$, and further the bend is expressed by a cubic equation between $z=0$ and $X$,

$$
y=-\frac{2 d}{X^{3}} z^{3}+\frac{3 d}{X^{2}} z^{2} \quad(0 \leq z \leq X)
$$

For type a bending (Figure 3a),

$$
\Delta U_{\mathrm{intra}}^{\mathrm{a}}=\frac{k}{2} \sum_{i}\left(y_{i+3}-y_{i+2}-y_{i+1}+y_{i}\right)^{2}
$$

where $y_{i}$ is the ordinate of the $i$-th unit and $k$ the force constant for the torsion of the $\mathrm{C}-\mathrm{C}$ bond. The value of $k$ can be obtained from the potential of hindered rotation of the $\mathrm{C}-\mathrm{C}$ bond as $k=4.11 \times 10^{3} \mathrm{dyn} / \mathrm{cm} .^{7}$ By approximating the chain as a one-dimensional elastic continuum with low curvature $\left(y_{i+1}-y_{i} \ll c / 2\right)$, eq 4 is reduced to

$$
\Delta U_{\mathrm{intra}}^{\mathrm{a}}=\frac{2 k}{\rho^{3}} \int_{0}^{X}\left(\frac{\mathrm{d}^{2} y}{\mathrm{~d} z^{2}}\right)^{2} \mathrm{~d} z=\frac{24 k d^{2}}{\rho^{3} X^{3}}
$$

In the case of type $b$ bending (Figure $3 b$ ),
$\Delta U_{\mathrm{intra}}^{\mathrm{b}}=\frac{K}{2} \sum_{i}\left(\sqrt{\left(y_{i+1}-y_{i-1}\right)^{2}+\left(z_{i+1}-z_{i-1}\right)^{2}}-c\right)^{2}$

where the force constant $K$ is due to bond-angle variation. The value of $K$ is calculated by use of the Urey-Bradley potential from force constants of $\mathrm{C}-\mathrm{C}-\mathrm{C}$ bond-angle variation and distance variation between unbonded carbons as $1.17 \times 10^{5} \mathrm{dyn} / \mathrm{cm}^{8}{ }^{8}$ In the continuum approximation,

$$
\Delta U_{\mathrm{intra}}^{\mathrm{b}}=\frac{K}{2 \rho} \int_{0}^{X}\left(\frac{\mathrm{d} y}{\mathrm{~d} z}\right)^{4} \mathrm{~d} z=\frac{K}{2 \rho} \frac{(6 d)^{4}}{630 X^{3}}
$$

For type $\mathrm{c}$ bending which is a composite of the above two (Figure 3c), the energy is simply additive because deformations of types $a$ and $b$ belong to different normal modes of vibrations in the polymethylene chain. The result is given for the bending to $45^{\circ}$ to the zigzag plane by

$$
\begin{aligned}
\Delta U_{\text {intra }}^{\mathrm{c}} & =\frac{1}{2} \Delta U_{\mathrm{intra}}^{\mathrm{a}}+\frac{1}{4} \Delta U_{\mathrm{intra}}^{\mathrm{b}} \\
& =\left[\frac{12 k d^{2}}{\rho^{3}}+\frac{K}{8 \rho} \frac{(6 d)^{4}}{630}\right] \frac{1}{X^{3}}
\end{aligned}
$$

The three quantities, $\Delta U_{\text {intra }}^{\mathrm{a}}, \Delta U_{\text {intra }}^{\mathrm{b}}$, and $\Delta U_{\text {intra }}^{\mathrm{c}}$, are all proportional to $X^{-3}$ and numerical evaluation of the proportional constants with $d=l^{*} / 2$ (shift by one-half of chain distance) 
leads to the following approximate equations

$$
\Delta U_{\text {intra }}^{\mathrm{b}} \simeq 4 \Delta U_{\text {intra }}^{\mathrm{a}}, \quad \Delta U_{\text {intra }}^{\mathrm{c}} \simeq 1.5 \Delta U_{\text {intra }}^{\mathrm{a}}
$$

The average intrachain potential $\Delta \bar{U}_{\text {intra }}$ for $d=$ $l^{*} / 2$ may be taken therefore as

$$
\Delta \bar{U}_{\text {intra }}=2 \Delta U_{\text {intra }}^{\mathrm{a}}
$$

It must be emphasized that the bending of a chain has been hitherto assumed to be caused by a small elastic deformation around the transconformation and not by trans-gauche transformation. The latter will be discussed in detail later in this paper.

\section{CHAIN BENDING AROUND CHAIN END}

In this section, a crystal of infinite lamella thickness is considered. When a chain has an end in the crystal, a vacancy row forms from the end to the lamella surface if chains surrounding the chain with the end remain linear. However, the increase in interchain energy by the vacancy row makes a neighbor chain or chains bend to fill up the vacancy.

There are in principle several ways in which such a bend may form but the following two extremes may be immediately eliminated because they possess higher strain energy than the original vacancy. (1) Six neighbor chains approach the vacancy isotropically. In this case, the strong repulsion potential between chains, or the van der Waals radius of chains, makes such deformation impossible. (2) A single neighbor chain bends to fill up the vacancy (Figure 4a). In this case, the vacancy moves to a neighboring position and the total potential increases by the intramolecular contribution due to bending. The case whereby chains successively bend till the crystal edge or another chain end of opposite sense is reached may be possible only when these are very close to the original chain end (Figures $4 \mathrm{~b}$ and $\mathrm{c}$ ). In this instance, dipole screw dislocations with opposite Burgers vectors (Burgers vector, $l^{*}$ ) run between the end and the crystal edge, or between two ends. The bend shift of chains is $d=l^{*}$ (not $l^{*} / 2$ ) and hence the increase in intramolecular potential which is proportional to $d^{2}$ or $d^{4}$ (see eq 5 and 7) may be high except in the case where the screw dislocations are sufficiently short.

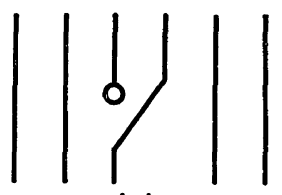

(a)

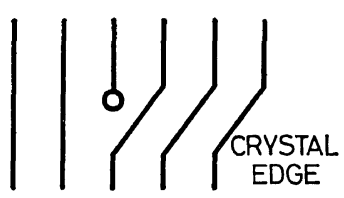

(b)

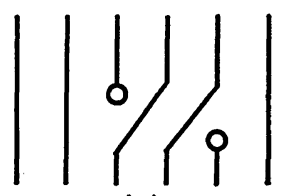

(c)

Figure 4. Chain bending with the shift $d=l^{*}$ : (a), bending of a neighboring chain; (b), successive bending to the crystal edge; (c), successive bending to a neighboring end of opposite sign.

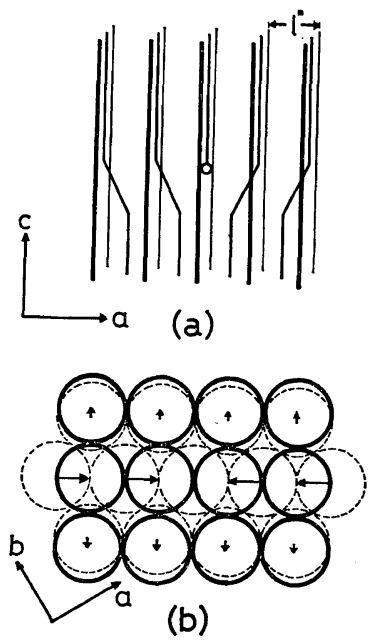

Figure 5. Chain bending with the shift of $d=l^{*} / 2$ in the plane with a chain end. Chains in two neighboring planes are slightly displaced. Dashed and solid circles represent chains above and below chain end, respectively.

Apart from the special cases mentioned above, therefore, neighboring chains on both sides of the chain end will bend with the shift by $d=l^{*} / 2$ to fill up the vacancy row. In this case, if the bend occurs only in the plane with the end and 
chains in neighboring planes remain linear, the crystal is transformed from hexagonal symmetry into tetragonal as illustrated in Figure 5, resulting in a marked increase in interchain potential. Consequently, chains in neighboring planes will bend also by $l^{*} / 2$, making a pair of screw dislocations through the chain end. ${ }^{2}$ Two cases are then possible. When the shift of neighboring planes is in the opposite direction as illustrated in Figure 6, the screw dislocations have a Burgers vector of the same sign. On the other hand,
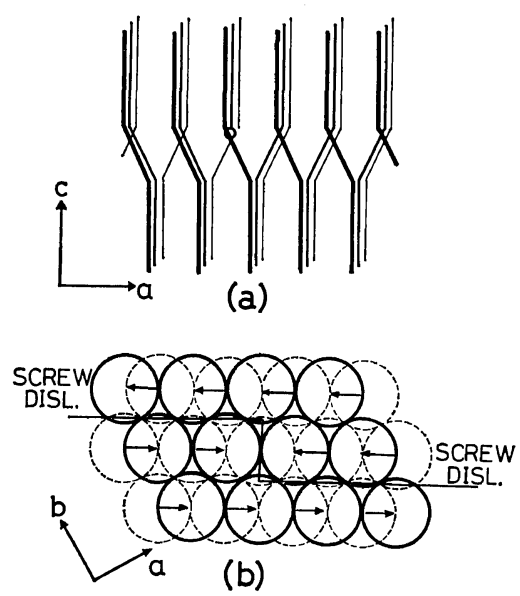

Figure 6. Jogged screw dislocations caused by chain bending around the vacancy row. Top represents view from [110]-axis, bottom from $c$-axis. Dashed and solid circles represent chains above and below chain end, respectively.

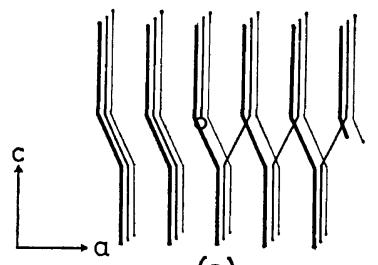

(a)

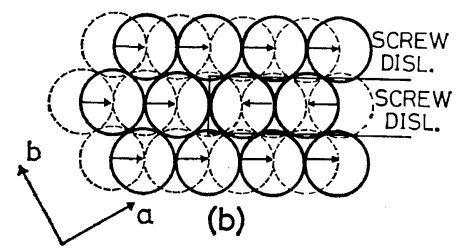

Figure 7. Coupled screw dislocation caused by chain bending around the vacancy row. Expressions are the same as in Figure 6. when the shift is in the same direction as shown in Figure 7, the screw dislocations have a Burgers vector of the opposite sign. In the following, the former will be called jogged screw dislocations and the latter coupled screw dislocations.

Simple calculations on the basis of intermolecular and intramolecular potentials support the above conclusion that a pair of screw dislocations are the most stable defects caused by a chain end except in special instances. Edge dislocations proposed by Predecki and Statton ${ }^{2}$ as one of the possible dislocations caused by chain ends are concluded to have a higher energy than the original vacancy row for a hexagonal crystal. For nylon crystals, however, approximated by a tetragonal lattice, the above conclusion should be modified.

\section{STRAIN ENERGY OF SCREW DISLOCATION}

As has been described in the preceding section, the increase in intramolecular potential depends on the length of bending, $X$. First we consider the energy of a subsystem consisting of seven chains which constitute a hexagonal unit after screw dislocation has occured (Figure 8).

For calculating the interchain potential, two chains 1 and 7 are assumed to bend by $d=l^{*}$ relatively to other chains. The curved parts of chains 1 and 7 are expressed therefore from eq 3 by

$$
\left.\begin{array}{l}
y^{(1)}=-\frac{2 l^{*}}{X_{e}{ }^{3}} z^{3}+\frac{3 l^{*}}{X_{e}{ }^{2}} z^{2}=-l^{*}\left(2 \xi^{3}-3 \xi^{2}\right), \\
y^{(7)}=-\frac{2 l^{*}}{X_{e}{ }^{3}} z^{3}+\frac{3 l^{*}}{X_{e}{ }^{2}} z^{2}-l^{*}=-l^{*}\left(2 \xi^{3}-3 \xi^{2}+1\right),
\end{array}\right\}
$$

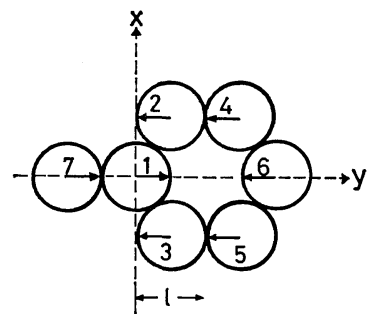

(a)

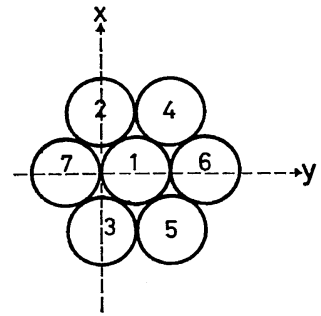

(b)
Figure 8. Subsystem of seven chains around a vacancy row: (a), before; (b), after bending by $d=l^{*} / 2$, respectively. 
where $\xi=z / X_{e}$ and the suffix $e$ refers to the value in the vicinity of the chain end.

The increase in interchain potential may be the sum of two terms, $\Delta U_{\text {inter }}^{(1)}$ between chain 1 and other chains and $\Delta U_{\text {inter }}^{(7)}$ between chain 7 and other chains. The increase in interchain potential between chain 1 and chains 2 to 7 is given from eq 2 as

$$
\begin{aligned}
\Delta U_{\mathrm{inter}}^{(1)}= & \sum_{i=2}^{7} \int_{0}^{X_{e}}\left[-\frac{3 \pi \rho \varepsilon}{4}\left(\frac{r^{* 6}}{l_{1 i}^{5}}-\frac{21}{64} \frac{r^{* 12}}{l_{1 i}^{11}}\right)\right] \rho \mathrm{d} z \\
& -\sum_{i=2}^{7}\left[-\frac{3 \pi \rho \varepsilon}{4}\left(\frac{r^{* 6}}{l_{1 i}^{* 5}}-\frac{21}{64} \frac{r^{* 12}}{l_{1 i}^{* 11}}\right) \rho X_{e}\right]
\end{aligned}
$$

where $l_{i j}$ represents the distance between chain $i$ and chain $j$ after bending and $l_{i j}^{*}$ that before bending,

$$
\left.\begin{array}{l}
\left.l_{12}^{2}=l_{13}^{2}=\left[l^{*}\left(2 \xi^{3}-3 \xi^{2}\right)+\frac{1}{2} l^{*}\right]^{2}+\left(\frac{\sqrt{3}}{2} l^{*}\right)^{2}\right) \\
l_{14}^{2}=l_{15}^{2}=\left[l^{*}\left(2 \xi^{3}-3 \xi^{2}\right)+\frac{3}{2} l^{*}\right]^{2}+\left(\frac{\sqrt{3}}{2} l^{*}\right)^{2} \\
l_{16}^{2}=\left[l^{*}\left(2 \xi^{3}-3 \xi^{2}\right)+2 l^{*}\right]^{2} \\
l_{17}=l^{*} \\
l_{1 i}^{*}=l^{*} \quad(i=2,3, \ldots, 7)
\end{array}\right\}
$$

The increase in interchain potential between chain 7 and chains 1 to 6 is safely approximated by that between 7 and 1 to 3 and is expressed in terms similar to eq 12 as

$$
\begin{aligned}
& \Delta U_{\text {inter }}^{(7)}=\sum_{i=1}^{3} \int_{0}^{X}\left[-\frac{3}{4} \pi \rho \varepsilon\left(\frac{r^{* 6}}{l_{7 i}^{5}}-\frac{21}{64} \frac{r^{* 12}}{l_{7 i}^{11}}\right)\right] \rho d z \\
& -\sum_{i=1}^{3}\left[-\frac{3}{4} \pi \rho \varepsilon\left(\frac{r^{* 6}}{l_{7 i}^{* 5}}-\frac{21}{64} r^{* 12} l_{7 i}^{* 11}\right) \rho X_{e}\right]
\end{aligned}
$$

where

$$
\left.\begin{array}{l}
l_{71}=l^{*} \\
l_{72}^{2}=l_{73}^{2}=\left[l^{*}\left(2 \xi^{3}-3 \xi^{2}+1\right)+\frac{1}{2} l^{*}\right]^{2}+\left(\frac{\sqrt{3}}{2} l^{*}\right)^{2} \\
l_{71}^{*}=l_{72}^{*}=l_{73}^{*}=l^{*}
\end{array}\right\}
$$

The increase in intrachain potential of seven chains is given under the approximation of average force constant of bending (eq 10),

$$
\Delta U_{\mathrm{intra}}=\frac{84 k l^{* 2}}{\rho^{3} X_{e}^{3}}
$$

The total increase in potential energy is thus obtained as

$$
\Delta U=\Delta U_{\text {inter }}^{(1)}+\Delta U_{\text {inter }}^{(7)}+\Delta U_{\text {intra }}
$$

$X_{e}$ can be determined by the condition $\Delta U=$ minimum with respect to $X_{e}$ as

$$
X_{e}=\left(1.58 \times 10^{2} \frac{l^{*}}{\rho} \frac{k}{\varepsilon}\right)^{1 / 4} \frac{1}{\rho}
$$

The numerical value of $X_{\theta}$ for polyethylene is $14.7 \AA$ which corresponds to $11.6 \mathrm{CH}_{2}$ units.

When the screw dislocation forms, the intersect of chains in two adjacent planes is aligned along the dislocation line (Figures 6 and 7). The length of bend $X_{i}$ in this region is calculated in a way similar to the above. $X_{i}$ is proportional to $\left(l^{*} k / \rho \varepsilon\right)^{1 / 4} / \rho$ in the same way as $X_{e}$ but proportional constants differ from $X_{e}$ and also between the jogged and coupled forms. $X_{i}=24.4 \AA$ for the former and $20.5 \AA$ for the latter. This difference comes from the different arrangement of chains in the two types of dislocations. In the case of coupled form, a chain between a pair of dislocations has an intersect with chains on both sides (Figure 7) but in the case of the jogged form, on the other hand, the chain near the dislocation has an intersect with a chain on one side (Figure 6).

The length of bend $X$, therefore, is equal to $X_{e}$ at the immediate vicinity of chain ends, and increases gradually towards $X_{i}$ with increasing distance from the end.

The above estimation of $X$ and corresponding potential energy leads to the expression of the total energy increase due to a screw dislocation, $\Delta U$ (screw), as

$$
\left.\begin{array}{rl}
\Delta U(\text { screw }) & =\Delta U_{\text {inter }}(\text { screw })+\Delta U_{\text {intra }}(\text { screw }) \\
\Delta U_{\text {inter }}(\text { screw }) & =1.73 \pi \rho^{2} \varepsilon l^{*} X_{e}+0.060 \pi m \rho^{2} \varepsilon l^{*} X_{i} \\
\Delta U_{\text {intra }}(\text { screw }) & =\frac{12 n k l^{* 2}}{\rho^{3} X_{i}{ }^{3}}
\end{array}\right\}
$$

where $n$ is the number of the bent chains per chain end or, in macroscopic terms, the number of chains in the strained range around a screw dislocation, and $m$ is the number of chain intersects along a screw dislocation. The average value of $n$ in a crystal can be estimated from chain end density but $m$ depends on the distribution of chain ends and screw dislocations in the crystal. Since the Burgers vector is smallest 
for partial dislocations in the $\langle 110\rangle$ direction in polyethylene, the screw dislocation may run mostly on $\{110\}$ planes and the dislocation from one chain end may run to another chain end which is positioned in the vicinity of the line drawn from the former end in $\langle 110\rangle$ directions. The value of $m$ depends on the effective range of interaction around this line. Screw dislocations by two chain ends may be coupled through a jog (a short edge dislocation) when the ends lie within the range but may not be coupled outside the range. In the following treatment, the range is assumed to be five planes and $m$ is taken as $n / 5$ as a reasonable estimate.

In an orthorhombic crystal of polyethylene in which $l^{*}$ corresponds to the displacement of $(a / 2, b / 2,0)$, the screw dislocation in the $\{110\}$ planes is partial and the stacking fault forms with the dislocation. In the present treatment in which a chain is approximated by a linear arrangement of units for interchain potential, the estimation of energy due to the stacking fault is not taken into account.

As can be readily seen from eq 18 , the length of the bend measured by the number of units, $\rho X$, is proportional to $\left(l^{*} k / \rho \varepsilon\right)^{1 / 4}$. Since the Young's modulus $E_{||}$parallel to the chain axis is proportional to $K / \rho l^{* 2}$ and the modulus $E_{\perp}$ perpendicular to the axis is proportional to $\rho^{2} \varepsilon / l^{*}, \rho X$ is proportional to $\left(\rho l^{*}\right)^{1 / 2} \times\left(E_{||} / E_{\perp}\right)^{1 / 4}$. In other words, the strain field in the polymer crystal due to a chain end or an intersect has a larger extension along the chain axis than along the perpendicular axis, the ratio being proportional to $\left(E_{||} / E_{\perp}\right)^{1 / 4}$. This conclusion may be also predicted from the continuum theory of elasticity.

In the dislocation theory, it is often convenient to divide the strain energy into two parts: core energy and the elastic energy of the long-range strain field. In the present treatment, however, the energy was calculated at a molecular level and hence this differentiation was not necessary. The strain field was assumed to be limited within the layer of thickness $X_{i}$ along the chain axis and to extend to the nearest neighboring dislocations in the plane perpendicular to the chain axis. $X_{i}$ was found to be smaller for the coupled screw dislocation than for the jogged one. This indicates the strain field is less extended in the coupled form.

\section{CRITERION OF FORMATION OF SCREW DISLOCATION}

In the preceding sections, the crystal was assumed to have an infinite thickness and the effect of lamella surface was not considered. In the real crystal with a finite thickness, however, a vacancy row from the chain end to the lamella surface has a finite length and hence increase in interchain potential due to the vacancy row, $\Delta U(\mathrm{vac})$, is also finite. The screw dislocation may form only under the condition

$$
\Delta U(\mathrm{vac})>\Delta U(\text { screw })
$$

$\Delta U$ (vac) is proportional to the distance $Z$ of the chain end from the lamella surface,

$$
\Delta U(\mathrm{vac})=10.7 \rho^{2} \varepsilon l^{*} Z
$$

The critical value of $Z$ for screw dislocation formation is calculated for polyethylene from eq 19 and 21 to be $Z_{c}=30 \AA$ for $n=100$. This $n$ value is derived under the assumptions that the number-average degree of polymerization is $2 \times 10^{4}$, one-tenth of the whole chain ends are effective to screw dislocation formation and the lamella thickness is $100 \AA$. When $Z$ is smaller than $Z_{c}$, the vacancy row is the most stable defect caused by a chain end, which is equivalent to a pair of edge dislocations with Burgers vectors of opposite sign (Figure 9).

In the case of polyoxymethylene in which the packing of molecules is exactly hexagonal $\left(l^{*}=\right.$ $4.46 \AA)^{9}$ and $E_{||}$is lower but $E_{\perp}$ is higher than

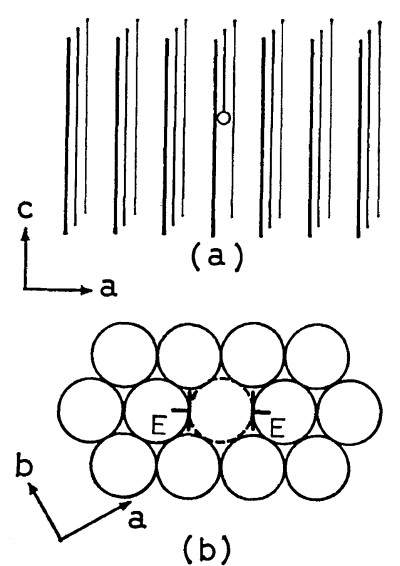

Figure 9. Vacancy row or a pair of edge dislocations caused by a chain end. Top represents view from [110]-axis and bottom from $c$-axis. 
polyethylene, ${ }^{10}$ the value of $X$ may be smaller than polyethylene and consequently $Z_{c}$ may be also smaller.

Calculations for poly(oxymethylene) are carried out in a nanner essentially similar to polyethylene. For intrachain potential, however, molecules are assumed to be a circular rod form with the same second moment of cross section as polyethylene. This assumption is reasonable because $l^{*}$ is almost the same in the two polymers. It is further assumed that, for polyethylene, the bending of the rod is equivalent to type $b$ bending because $E_{||}$is related to the bond-angle variation. Under these assumptions, the calculation for polyethylene can be reduced to that for poly(oxymethylene) by use of $E_{||}$and $E_{\perp}$ as parameters. The calculated values for poly(oxymethylene) are $X_{e}=$ $10 \AA, X_{i}=15 \AA$ (jogged type) and $Z_{c}=20 \AA$ for the same chain-end density and the same lamella thickness as polyethylene.

\section{CHAIN BENDING ACCOMPANYING TRANS- GAUCHE TRANSFORMATION}

Chain bending of polyethylene which has so far been discussed has been limited to elastic deformation of chains around trans-conformation. In this section, bending accompanying transgauche transformation will be discussed.

In Figure 10, an example with the least gauche bonds is illustrated, in which the shift perpendicular to the chain axis is almost equal to $l^{*}$ along the $b$-axis. Such a form of bending might occur for filling up a vacancy row by the bending of a single neighboring chain and hence, as described in the previous section, the bending only increases the intrachain potential. The increase in intrachain energy in Figure 10 is obtained as $11.2 \times 10^{-14} \mathrm{erg}$ on the simple assumption that one gauche conformation has excess energy of $5.6 \times 10^{-14} \mathrm{erg}$. The Boltzmann factor for this bending is $\exp \left(-\Delta U_{\text {intra }} / k T\right)=$ $0.067\left(T=300^{\circ} \mathrm{K}\right)$ when the interchain energy is ignored. Such a form of bending, therefore, may be thermally excited at an appreciable frequency. Hoffman, Williams, and Passaglia ${ }^{11}$ have attempted to interpret the dielectric $\gamma$-relaxation of poly(trifluorochloroethylene) in terms of this type of motion.

Figure 11 illustrates two examples of bending

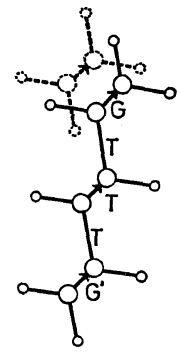

(1)

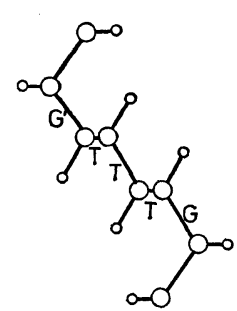

(2)
Figure 10. Chain bending of polyethylene chain with trans-gauche transformation with the shift $d \simeq l^{*}$. Two gauche bonds are included: (1) view from $c$-axis (dashed figure represents regular position); (2) view from $a$-axis.
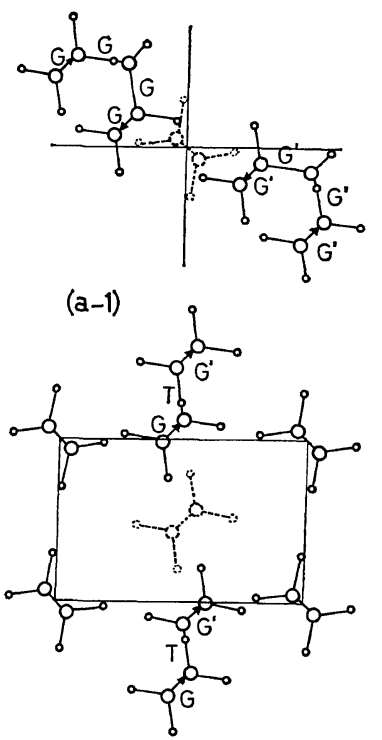

(b-1)

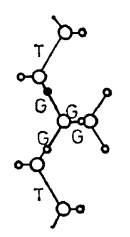

$(a-2)$

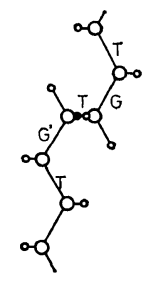

(b-2)
Figure 11. Two types of chain bending ( $a$ and $b$ ) with trans-gauche transformation with the shift $d \simeq l^{*} / 2$ : (a-1) and (b-1), view from $c$-axis; (a-2), view from [110]0]; (b-2), from $a$-axis. (a) and (b) include four and two gauche bonds, respectively. Shift by bending along $c$-axis is approximately $c$ and $c / 2$ for (a) and (b), respectively.

accompanying trans-gauche transformation with the shift $\mathrm{d}$ approximately equal to $l^{*} / 2$. As has been described in the previous section, this type of bending might be effective for screw dislocation formation if the energy is lower than elastic bending. Calculations reveal, however, this is not the case and hence trans-gauche transfor- 
mation may not be expected in the screw dislocation.

The bending in Figure 11b was first proposed by Pechhold and called "kinked chain". ${ }^{12}$ Pechhold calculated the increase in intrachain energy alone but the addition of the increase in interchain potential may make the energy appreciably higher and the probability of thermal excitation of kinked chains may be lower than Pechhold estimated.

\section{INTERACTION AMONG CHAIN ENDS}

As has been described in the previous section, a chain end in the crystal generates screw dislocations or a vacancy row. When the screw dislocation forms, defects caused by another chain end are strongly affected by the strain field of the dislocation. In the following, defects by two chain ends are discussed two-dimensionally in the plane, including the dislocation. Two cases are then possible.

In the first case, the two chain ends are well fitted so that two screw dislocations from each end join together (Figure 12). As has been already pointed out by Predecki and Statton, ${ }^{2}$ if two chain ends have opposite sign, in other words, one from the top and the other from the bottom, a dipole screw dislocation with opposite Burgers vectors forms. On the other hand, if two chain ends have the same sign, both from the top or both from the bottom, screw dislo- cations of the same Burgers vectors run through the ends with jogs (or short edge dislocations) at the chain ends.

In the second case where two chain ends are not well fitted, a vacancy row is created with screw dislocations. Figure 13 represents three possible cases: (a) a chain end located on coupled screw dislocations which is caused by another chain end beyond the figure, (b) a chain end located on a jogged screw dislocation, and (c) a chain end located apart from the dislocation which is not depicted in the figure. Among three possible cases in Figure 13, case a (coupled screw dislocations with a vacancy row) is proved unstable because the activation energy for vacancy motion from (a) to $\left(a^{\prime}\right)$ is small and the state (a) goes thermally to the state in Figure 12 1a. Cases $b$ (jogged screw dislocations with a vacancy row) and c (a vacancy row only) are, on the other hand, metastable because the activation energy for vacancy motion is very high.

We now consider a three-dimensional crystal. If the vacancy row formed as in Figures $13 \mathrm{~b}$ and $\mathrm{c}$ has sufficient length to the crystal surface, chains on a molecular plane other than the original dislocation plane bend to fill up the vacancy. If the initial screw dislocation is on the (110) plane, the new dislocation may be on (1110) plane or vice versa. As a consequence, a dislocation network is extended within the crystal and the crystal has a mosaic structure. ${ }^{13}$

Various physical properties of polymer crystals

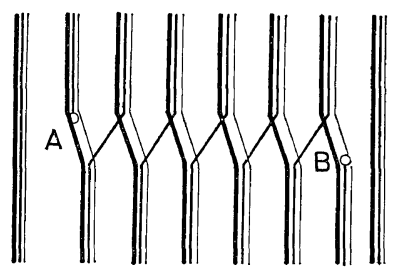

(1a)

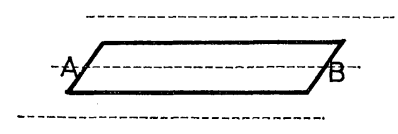

(1b)

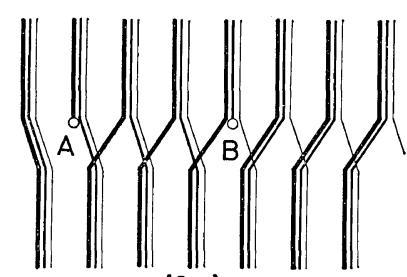

(2a)

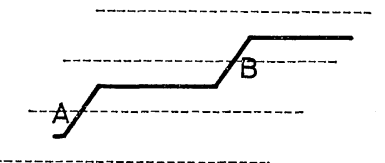

(2b)

Figure 12. Screw dislocations by two chain ends positioned in the same or adjacent planes: (1a) and (1b), coupled or dipole screw dislocations; (2a) and (2b), jogged screw dislocations. 


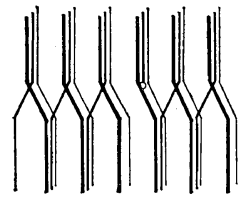

(a)

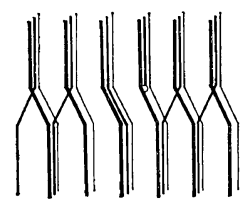

(a)

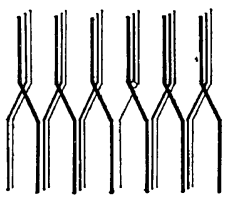

(b)

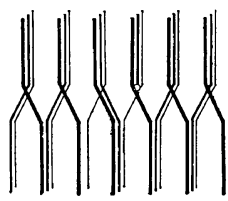

(b')

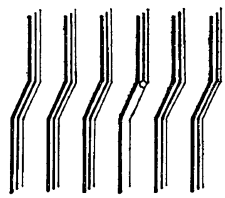

(c)

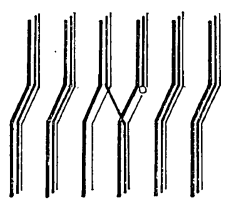

(c)

Figure 13. Three possible cases of defects by two chain ends which are not well fitted with each other: (a), coupled screw dislocations with a vacancy row; (b), jogged screw dislocations with a vacancy row; (c), a vacancy row alone. One end generating screw dislocations is beyond the figure. $\left(a^{\prime}\right),\left(b^{\prime}\right)$, and $\left(c^{\prime}\right)$, represent the case the vacancy row moves to an adjacent position.

may be attributable to defects caused by chain ends. A lower density of polyethylene single crystals than expected from unit cell dimensions ${ }^{14}$ may be partly ascribed to vacancy rows. In the following, plastic deformation and anelastic behavior of polyethylene crystals will be interpreted in terms of vacancy rows and screw dislocations.

\section{INTERPRETATION OF PHASE TRANSFOR- MATION AND TWINNING IN TERMS OF VACANCY ROWS AND SCREW DISLOCATIONS}

According to Kiho, et al. ${ }^{3}$ who studied plastic deformation of [polyethylene single crystals on the substrate, two mechanisms take place at an early stage of deformation: phase transformation from orthorhombic lattice to monoclinic and $\{110\}$ twinning. When the crystal is deformed along the $b$-axis, only the transformation is observed. When the crystal stretches along the $a$-axis, on the other hand, the twinning is predominant. For deformation perpendicular to the growth surface $\{110\}$ or parallel to it, both mechanisms coexist. These mechanisms will be interpreted in terms of vacancy rows and screw dislocations, which have been proved in the previous sections to be the most stable defects generated by chain ends in as-grown crystals.

Now we consider a $\{110\}$ plane including a vacancy row. When the stress is applied and increases the interchain potential, the vacancy row will become less stable and neighboring chains on both sides of the vacancy will bend to fill it up. In this case, chains are forced to bend with a smaller $X$ than usual. One set of chains having a zigzag plane almost perpendicular to the $\{110\}$ plane can produce type a bending but the other set of chains with a zigzag plane almost parallel to the $\{110\}$ plane might produce type $b$ bending which requires high energy (Figure 2). If the latter set of chains produces bending accompanying $90^{\circ}$ rotation around the chain axis (twist-bending) instead of type $b$ bending, in the former of which the force constant is relevant to the internal rotation of carbon-carbon bond, the molecular arrangement around the vacancy row will form a monoclinic lattice. For formation of the monoclinic phase, a chain adjacent to the vacancy must shift along the $c$-axis by $c / 2$, which is required for good fitting among chains. Occurrence of such transformation at various places in the crystal is expected because of the existence of many vacancy rows in the crystal, resulting in phase transformation.

Possibility of twist-bending for small $X$ is proved in the following. The intramolecular potential for twist-bending is the sum of type a bending and twist. The intramolecular potential for twist by $90^{\circ}$ is written as 


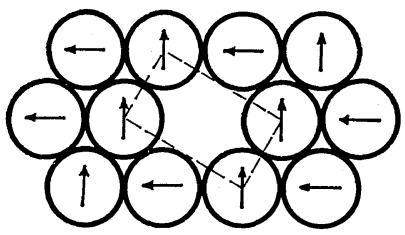

(a)

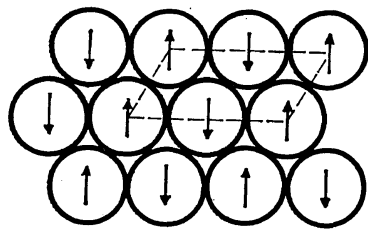

(b)

Figure 14. Transformation of (a) orthorhombic phase to (b) monoclinic caused by type a and twist bending around a vacancy row in polyethylene.

$$
\begin{aligned}
\Delta U_{\mathrm{intra}}^{t} & =\frac{k^{\prime}}{2} \Sigma\left(\psi_{i+1}-\psi_{i-1}+\psi_{i}-\psi_{i-2}\right)^{2} \\
& =\frac{2 \pi^{2} k^{\prime}}{\rho X}=\frac{2 \pi^{2} k a^{2}}{\rho X}
\end{aligned}
$$

where $\phi_{i}$ is the angular displacement of the $i$-th carbon atom from the zigzag plane around the chain axis, $k^{\prime}$ the torsional force constant $\left(k^{\prime}=\right.$ $\left.k a^{2}\right), a$ the distance of the carbon atoms from the chain axis and $X$ the length of the twisted part of a chain taken as being equal to the length of the bending (Figure 15). As shown in Figure 16, twist-bending has a higher energy than type $b$ bending for large $X$ but becomes more probable than type $\mathrm{b}$ bending for small $X(<13 \AA)$. Increase in the intermolecular potential by the applied stress makes the length of the bending $X$ short and twist-bending is expected to occur more easily.

This mechanism can interpret the partial phase recovery from the monoclinic to the orthorhombic lattice when the applied stress is removed.

As has been described in the previous section, chains in two successive $\{110\}$ planes intersect each other along the screw dislocation line, forming a stacking fault. When shear stress is applied in the $\{110\}\langle 1 \overline{1} 0\rangle$ direction, a slip by $(a, b, 0)$ occurs between the $\{110\}$ planes including

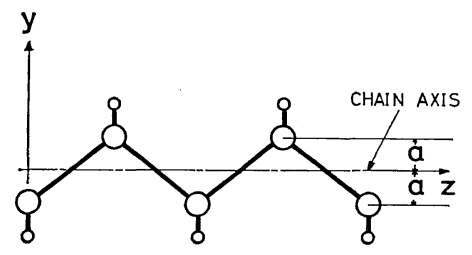

(a)

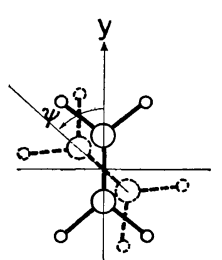

(b)
Figure 15. Twist of a planar zigzag chain around the chain axis.

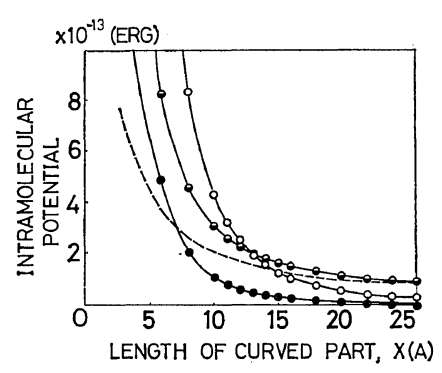

Figure 16. Intramolecular potential for various types of bending with the shift of $l^{*} / 2$ plotted against length of curved part: 0 , type a bending; $\bigcirc$, type $b$ bending; $\odot$, twist-bending (twist by $\pi / 2$ ). Broken line represents twist without bending (twist by $\pi / 2$ ).

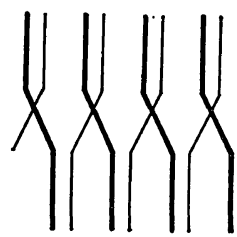

(a)

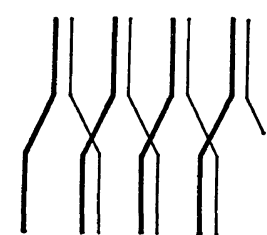

(b)
Figure 17. Inversion of intersect along a screw dislocation by applied shear stress: (a), original intersect; (b), after inversion.

a screw dislocation, making the sign of intersection of chains opposite (Figure 17). In this process, the original partial dislocation with the Burgers vector $\boldsymbol{l}^{*}$ changes its Burgers vector to $-l^{*}$ and, in addition, two partial dislocations with the Burgers vector $l^{*}$ are created in parallel on both sides of the original one. Layers of stacking faults are thus piled up, resulting in the $\{110\}$ twin against the original lattice (Figure 18). 


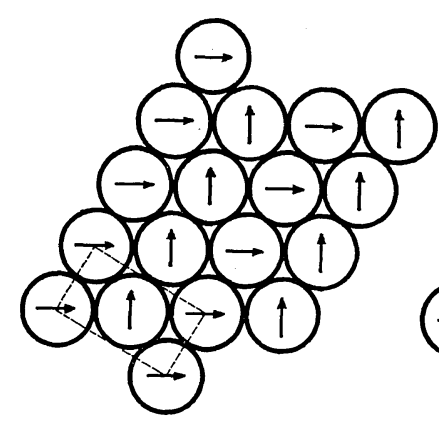

(a)

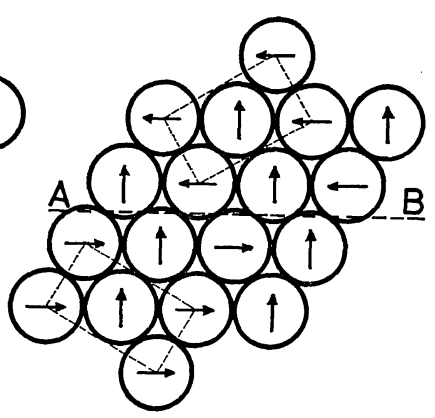

(b)

Figure 18. [110] Twinning of polyethylene caused by cooperative slips making opposite the sign of intersects along the screw dislocations: (a), original lattice, (b), lattice after cooperative slips on each plane above the plane $A B$ accompanied with shift along $c$-axis by $c / 2$ of alternate chains.

\section{A DISLOCATION THEORY FOR THE $\gamma$-RELAXATION}

A highly crystalline polymer, polyethylene for example, generally exhibits $\gamma$-relaxation in dynamic mechanical measurements at low temperatures. In the case of polyethylene (PE), the $\gamma$ relaxation appears around $-120^{\circ} \mathrm{C}$ at $100 \mathrm{~Hz} .^{15}$ The $\gamma$-relaxation is observed also in dielectric measurements for polar polymers.

According to Arai, Yano, and Wada, ${ }^{4}$ who compared the $\gamma$-relaxations among as-grown PE single crystals, annealed, and $\mathrm{HNO}_{3}$-treated ones, the $\gamma$-relaxation is composed of two peaks, the low-temperature peak $\left(\gamma_{1}\right)$ and the high-temperature peak $\left(\gamma_{2}\right)$. Since the $\gamma_{2}$-peak vanishes with $\mathrm{HNO}_{3}$-treatment but the $\gamma_{1}$ does not, the former is attributed to defects on the surface of lamellae and the latter to defects inside the lamellae. In the case of polycrystalline samples, the $\gamma_{2}$-peak may be assigned to interlamella defects or amorphous phase. A similar conclusion has been reached from the effects of irradiation and swelling on the $\gamma$-peak of polyethylene ${ }^{5}$ and that of crystallinity on the $\gamma$-peak. ${ }^{11,16}$

Low temperature relaxations in simple, non polymeric crystals, $100^{\circ} \mathrm{K}$ peak $(10 \mathrm{kHz})$ in aluminum for example, have been attributed to motion of dislocation and called Bordoni-peak. ${ }^{17}$ A mechanism similar to the Bordoni-peak will be proposed here for the $\gamma_{1}$-peak in polymers.

The calculation of strain energy of defects in the previous section indicated that, for crystals including chain ends, screw dislocations are the most stable among various possible types of defects except the case where chain ends are very close to the lamella surface. A dislocation line runs from one chain end to another and a dislocation network forms in the crystal. Figure 19 illustrates schematially the dislocations, in which points $\mathrm{A}$ and $\mathrm{B}$ represent a chain end, respectively. In what follows, directions of the chain axis and the dislocation line are tentatively called $c$ - and $a^{\prime}$-axes, respectively.

In thermal equilibrium, the pinned dislocation
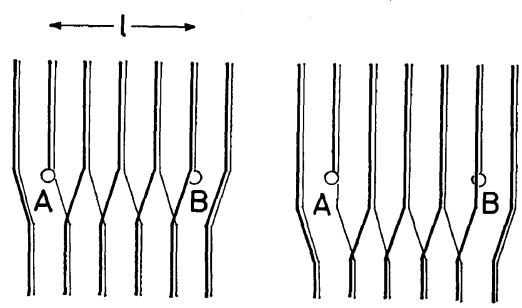

STATE 1

(a)
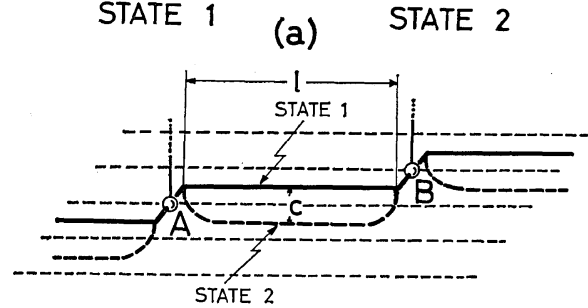

(b)

Figure 19. Schematic represention of motion of screw dislocations between two states. 


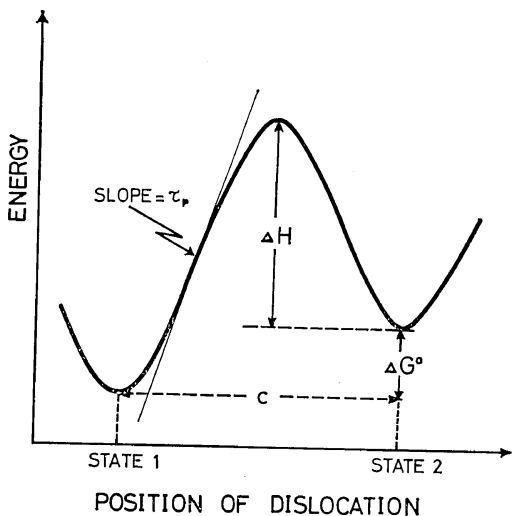

Figure 20. Energy map for motion of dislocation.

fluctuates among minimum energy positions over the Peierls potential, with a distribution determined by the free energy difference at each position. Points $\mathrm{A}$ and $\mathrm{B}$ serve as pinning points which are immobile during the motion of dislocation. When the dislocation moves downwards or upwards by a unit cell dimension $c$, a vacant site or an interstitial site forms at both $\mathrm{A}$ and $\mathrm{B}$, respectively. When the shear stress of $a^{\prime}$ direction in the $a^{\prime} c$-plane is suddenly applied to the crystal, the distribution should change with time accompanying the stress relaxation, but this requires a relaxation time.

We assume for simplicity (1) only two energy minima exist (two-state model in Figure 20), (2) the reaction is isothermal and only the strain changes with the reaction (volume relaxation). The relaxation strength may then be written, following the general theory of volume relaxation of the two-state model, ${ }^{18}$ as

$$
r=\frac{G(\infty)-G(0)}{G(\infty)}=\frac{G(0)}{k T} N(\Delta v)^{2} \frac{f K}{(1+K)^{2}}
$$

where $G(\infty)$ and $G(0)$ are instantaneous and equilibrium elastic moduli, respectively, $N$ the number of dislocations per unit volume, $k$ the Boltzmann constant, and $T$ the absolute temperature. $\Delta v$ represents deformation of the crystal when one dislocation moves from 1 to $2, f$ the dimensionless constant due to the orientation of dislocation with respect to the applied stress, and $K$ the equilibrium constant between the two states. $\Delta v=b c l$ and $N=1 / l L d$ where $b$ is the Burgers vector, $l$ the dislocation length, $c$ the unit cell length along the chain direction, $L$ the lamellae thickness, and $d$ the thickness of the range of interaction between a dislocation and a chain end which has been assumed to be five planes in estimating $m$ in eq 19. The equilibrium constant $K$ is expressed by $K=\exp \left(-\Delta G^{0} / R T\right)$ where $\Delta G^{0}$ is the free energy difference between the two-states.

The dynamic mechanical data for a single crystal mat of polyethylene ${ }^{15}$ clearly indicates $\gamma$-relaxation. The $\gamma_{1}$-peak which is attributed here to motion of dislocation is the one observed for an as-grown crystal before annealing. According to Sinnott's data ${ }^{15}$ for PE, the observed value of $r$ for $\gamma_{1}$-peak is 0.15 .

In calculating $r$ from eq 23, we must estimate the fraction of effective chain ends. Some of the chain ends are excluded from lamellae, and some others are included in the lamella but positioned close to lamella surface and hence make vacancy rows running from chain end to lamella surface. In the present case, we assume the fraction of chain ends which contribute to screw dislocation is one-tenth of all the ends. This leads to the dislocation length $l=40 \AA$ for polyethylene of number-average molecular weight $=$ $10^{5}$ and lamella thickness $=100 \AA$. The numberaverage and weight-average molecular weights of linear PE used by Sinnott ${ }^{15}$ were $1.1 \times 10^{4}$ and $2.3 \times 10^{5}$, respectively. Taking into account the fractionation in the crystallization process, we assume a major fraction of crystallites has a number-average molecular weight of $10^{5}$.

Using values $G(0)=2 \times 10^{10} \mathrm{dyn} / \mathrm{cm}^{2}, T=150^{\circ} \mathrm{K}$, $b=5 \AA$, and $c=2.5 \AA$, and assuming $f=1 / 3$ for isotropic orientation of dislocations in the polycrystalline specimen and $K=0.15$, we obtain from eq $23 r=0.15$, which agrees closely with the experimental value. This $K$ value corresponds to $\Delta G^{0}=0.7 \mathrm{kcal} / \mathrm{mol}$ which is reasonable as compared with the activation energy (Figure 20). $\Delta G^{0}$ might be estimated experimentally from the temperature dependence of $r$.

According to the present model, the activation energy $\Delta H$ of $\gamma_{1}$-relaxation is equal to the Perierls potential for the dislocation. The estimation of Peierls potential from a microscopic point of view is quite difficult even for a simple crystal. Here we use an approximate equation,

$$
\Delta H=\tau_{p} l b c
$$


where $\tau_{p}$ is the Peierls stress and is approximated by the yield stress extrapolated to $0^{\circ} \mathrm{K}$. Using the value of $\tau_{p}=5 \times 10^{8} \mathrm{dyn} / \mathrm{cm}^{2}, \Delta H$ is calculated from eq 24 as $10 \mathrm{kcal} / \mathrm{mol}$, which agrees closely with the observed value.

Some of the values used in the above calculation are rather tentative, but it must be emphasized that both the relaxation strength and the activation energy are predicted from the same numerical values which would seem to be reasonable for the real system.

\section{REFERENCES}

1. A. Keller and D. J. Priest, J. Macromol. Sci., B2, 479 (1968).

2. P. Predecki and W. O. Statton, J. Appl. Phys., 38, 4140 (1967).

3. H. Kiho, A. Peterlin, and P. H. Geil, J. Appl. Phys., 35, 1599 (1964).

4. K. Arai, O. Yano, and Y. Wada, Rep. Progr. Polym. Phys. Japan, 11, 267 (1968).

5. K. H. Illers, Kolloid-Z., 231, 622 (1969); Rkeol. Acta, 3, 185, 194, 202 (1964).

6. A. Odajima and T. Maeda, J. Polym. Sci., Part
C, 15, 55 (1966).

7. S. Mizushima, "Structure of Molecules and Internal Rotation," Academic Press, New York, N.Y., 1954.

8. M. Tasumi, T. Shimanouchi, and T. Miyazawa, J. Mol. Spectrosc., 9, 261 (1962); 11, 422 (1963).

9. H. Tadokoro, T. Yasumoto, S. Murahashi, and I. Nitta, J. Polym. Sci., 44, 266 (1960).

10. I. Sakurada, T. Ito, and K. Nakamae, J. Polym. Sci., Part C, 15, 75 (1966).

11. J. D. Hoffman, G. Williams, and E. Passaglia, J. Polym. Sci., Part C, 14, 173 (1966).

12. W. Pechhold, Kolloid-Z., 228, 1 (1968).

13. R. Hosemann, W. Wilke, and F. J. Balta Calleja, Acta Cryst., 21, 118 (1966).

14. A. Peterlin, J. Macrom. Sci., B3, 19 (1969).

15. K. M. Sinnott, J. Appl. Phys., 37, 3385 (1966).

16. R. W. Gray and N. G. McCrum, J. Polym. Sci., Part A-2, 7, 1329 (1969).

17. A. Seeger, "Handbuch der Physik" Vol. 7, Bd. 1, S. Flügge, Ed., Springer, Göttingen, 1955, p 383.

18. J. Lamb, "Physicla Acoustics," Vol. 2A, W. P. Mason, Ed., Academic Press, New York, N.Y., 196. 